\title{
Magnetic Flux Cancellation and Coronal Magnetic Energy
}

\author{
B.T. Welsch \\ Space Sciences Laboratory, University of California, Berkeley, CA 94720-7450; \\ welsch@ssl.berkeley.edu
}

\begin{abstract}
I investigate the processes at work in the cancellation of normal magnetic flux in solar magnetograms, and study the relationships between cancellation and the budget of free magnetic energy in the coronal magnetic field that can power solar flares and CMEs. After defining cancellation mathematically, I derive equations that quantify the evolution of free magnetic energy in response to arbitrary plasma flows on the boundary, including flows consistent with cancellation. While cancellation can reduce the magnetic energy in both the actual coronal field and the potential field matching the same normal field boundary condition, cancellation can, in the process, increase the difference between the two, i.e., cancellation can increase the free magnetic energy in the corona. By making simple assumptions based upon typical observed field configurations in filament channels, I show that cancellation tends to add free energy to these fields. Finally, I discuss the implications of this fact, as well as wider applications of the free energy flux formalism developed here. I also briefly address related issues, including the relationship of cancellation to Taylor's hypothesis.
\end{abstract}

Subject headings: Sun: activity - Sun: magnetic fields - Sun: coronal mass ejections (CMEs) - Sun: flares - MHD

\section{Introduction and Definitions}

Observers who first saw magnetic flux cancellation in magnetograms of the solar photosphere offered an operational definition of the phenomenon, "the mutual apparent loss of magnetic flux in closely spaced features of opposite polarity" (Livi et al. 1985).

Physically, Zwaan (1987) qualitatively explained this phenomenon in terms of magnetic reconnection. He identified three cases, which vary essentially only in the relative altitude of the reconnection site and the atmospheric layer imaged in the magnetograms: the reconnection could take place above, below, or at the magnetogram 
level. These "reconnective cancellation" scenarios are consistent with the observations of Harvey et al. (1999) that, in the quiet sun, cancelling magnetogram features typically originate in separate flux systems and coincide with coronal bright points. Harvey et al. (1999) argued that emerging U-shaped loops were far less common (if present at all) than submerging, inverted-U loops (or $\Omega$ loops) in their sample of reconnective cancellations, implying that the reconnection probably occurs above the photosphere. More recently, other observers have reported detailed signatures consistent with the magnetic reconnection implied by this process, also concluding that the reconnection takes place above the magnetograph's imaging layer; see, e.g., Chae (2003). Yurchyshyn and Wang (2001), however, reported the presence of upflows in a cancelling feature, inferred from Doppler shifts in the Ni $6767 \AA$ spectral line; accordingly, they placed the reconnection at the photosphere.

Other observervations, as well as MHD models, are consistent with the disappearance of vertical flux via essentially ideal processes, either the emergence of U-shaped field lines (van Driel-Gesztelyi et al. 2000; Magara et al. 2005) or the submergence of $\Omega$ loops (Chae et al. 2004). Van Driel-Gestelyi et al. (2000) claim to have observed the "clean" (essentially reconnection-free) emergence of a U-loop. Further, while MHD simulations by Fan (2001) implied that the bottoms of U-loops in an emerging, highly twisted flux tube might remain trapped beneath the photosphere, restrained by dense plasma trapped in the loop bottoms, more recent simulations by Magara et al. (2005) show that siphon flows can remove trapped mass from U-loops, allowing the loop bottom to emerge ideally, leading to cancellation. Using vector magnetograms, Chae et al. (2004) observed Doppler downflows in Stokes U and Q profiles between cancelling features, and noted that the rate of loss of vertical flux in the features matched the flux transport rate determined by the product of the horizontal magnetic field and downward velocity, consistent with ideal submergence. (If the submergence were diffusive, the plasma would not move downward at the same rate as the flux did; hence, the flux transport rate inferred by the Doppler velocity and horizontal field would not match the flux loss rate in the cancelling features.) Of course, the loops observed by Chae et al. (2004) might have formed non-ideally, by reconnection, prior to the observed submergence.

These varied observational and theoretical results suggest that each of these processes - reconnection, ideal emergence, and ideal submergence - could be at work on the Sun during cancellation, either singly or in combination. The prevalence of each process is an open question, and bears further study.

Flux cancellation processes have implications for space weather prediction. Cancella- 
tion is a necessary condition for the formation of prominences (Martin 1998), which are extended coronal emission features seen over the limb in chromospheric spectral lines (e.g., $\mathrm{H}-\alpha$ ). When the same structures are observed on the disk, they are seen as extended absorption features in chromospheric lines, and are known as filaments. Prominence eruptions, sometimes occurring over a few tens of coronal Alfvén crossing times, $\tau_{\text {cor }}$, are associated with coronal mass ejections, or CMEs (Forbes 2000), which are the primary drivers of severe space weather disturbances (Gosling 1993).

The detailed properties of prominence/filament systems are described elsewhere (Démoulin 1998; Martin 1998), but I briefly describe a few of their relevant properties here. They invariably form along polarity inversion lines (PILs), where the spatially averaged normal component of the photospheric magnetic field changes sign. Further, they only form along a particular subset of PILs known as filament channels (which exist independently of filaments) in which long, chromosheric H- $\alpha$ absorption features known as fibrils run parallel to the PIL, rather than across it. The fibril axes are thought to lie along the direction of the local magnetic field, which implies that the dominant field component in filament channels is parallel to the PIL. This is consistent with direct measurements of magnetic fields in prominence/filament systems performed by Bommier and Leroy (1998).

While the field topology in prominence/filament systems is unknown, Aulanier and collaborators $(2000,2003)$ applied flux rope models to photospheric magnetograms and were able to accurately reproduce the observed properties of several filaments a posteriori, and to predict the observed properties of a one filament a priori. In the flux rope models used by Aulanier et al. (2000,2003), the filament field lines are weakly twisted, by about one turn or less over the filament's length. In this work, I refer to field configurations with strong axial fields and weak or little twist as sheared arcades.

Models for prominence/filament formation can be broadly divided into two categories, those in which flux ropes emerge intact from beneath the photosphere (Rust and Kumar 1994; Low and Hundhausen 1995) or those in which weakly twisted flux ropes are formed by magnetic reconnection acting on fields that have already emerged (van Ballegooijen and Martens 1989; Martens and Zwaan 2001). Both types of model incorporate photospheric flux cancellation, as the emergence of U-loops in the former and submergence of post-reconnection, $\Omega$-loops in the latter.

An open question is how the coronal magnetic energy (henceforth, "energy" will refer to magnetic energy), $U_{M}=\int_{V} d V(\mathbf{B} \cdot \mathbf{B}) / 8 \pi$, evolves when flux cancels during prominence formation. While one would expect the emergence of a flux rope to 
increase the coronal energy, reconnective cancellation or the submergence of postreconnection loops can lower energy or remove it from the corona, so the question invites further study.

In addition to altering the energy in the actual magnetic field, $\mathbf{B}$, cancellation in an atmospheric layer $S$ also affects the energy of the potential magnetic field, $\mathbf{B}^{(P)}$, that matches the normal field boundary condition on B, i.e., $\left.B_{n}\right|_{\tilde{S}}=\left.B_{n}^{(P)}\right|_{\tilde{S}}$, where $\tilde{S}$ is the surface that bounds $V$. In the solar case, $V$ is the coronal volume, $\tilde{S}$ is the surface that encloses $V$, and $S$ is the region of $\tilde{S}$ on the bottom boundary of $V$. The potential field is current free, so $4 \pi \mathbf{J}^{(P)}=c\left(\nabla \times \mathbf{B}^{(P)}\right)=0$, implying $\mathbf{B}^{(P)}$ can be expressed as the gradient of a scalar potential, $\mathbf{B}^{(P)}=-\nabla \chi$. The potential field is of interest because it is the unique, minimum energy field that matches the same normal field boundary condition as B. Since the photosphere remains essentially unchanged on the rapid timescale of dynamic evolution of the coronal field $\left(\tau_{\mathrm{ph}} \gg \tau_{\text {cor }}\right)$ during a flare or CME, the energy available to drive such evolution is the free magnetic energy in the corona, $U_{F}=U_{M}-U_{M}^{(P)}$, where $U_{M}^{(P)}=\int_{V} d V\left(\mathbf{B}^{(P)} \cdot \mathbf{B}^{(P)}\right) / 8 \pi$. While the photospheric field can respond to changes in the coronal field - see, e.g., Sudol et al. (2004) - photospheric evolution is generally thought to be too slow to drive coronal restructuring on the coronal time scale.

The coronal field can store free magnetic energy in the form of large-scale, nonpotential fields in prominences/filaments for weeks (see, e.g., Démoulin 1998), because its evolution is constrained by its fixed topology: the corona's long length scales, high temperatures, and low densities mean that the field's nominal evolution is nearly ideal; equivalently, the magnetic Reynolds number is large. Consequently, while the coronal plasma possesses sufficient degrees of freedom to always reside in a minimum accessible energy state, the potential state is not always accessible. In the general case, $\mathbf{B}$ cannot relax to $\mathbf{B}^{(P)}$, the global energy minimum among the set of all magnetic fields that match the normal field boundary condition, $\left.B_{n}\right|_{\tilde{S}}$, but instead relaxes to a local energy minimum. Given the dominance of the Lorentz force over other terms in the momentum equation that describes the coronal field's evolution, the accessible minimum state, consistent with the field's topology, is presumably nearly force-free, $(\nabla \times \mathbf{B}) \times \mathbf{B} \simeq 0$, with $(\nabla \times \mathbf{B}) \simeq \alpha(\mathbf{x}) \mathbf{B}$

Even when the field's topology changes rapidly, via fast magnetic reconnection (perhaps due to the relatively brief occurrence of a spatially localized enhancement of magnetic diffusivity, manifested as a flare or sub-flare), the field is still unable to relax to the potential state, because the field's magnetic helicity (Berger and Field 1984) is approximately conserved (Berger 1984). (By definition, fields that possess gauge- 
invariant relative magnetic helicity are not potential.) Taylor (1985), suggested that the field relaxes instead to a linear, "constant- $\alpha$ " force-free state with approximately the same magnetic helicity as the field possessed prior to the onset of reconnection. Antiochos et al. (2002) have argued that the localized nature of reconnection in the solar case (in contrast to the global relaxation in the laboratory plasma experiments Taylor characterized), results in a variable- $\alpha$ (or non-linear) force-free field. Regardless of whether $\alpha$ varies in space or is constant, the presence of helicity in the post-reconnection field implies that the field is not potential.

It has been suggested (by, e.g., Low [2002] and others) that CMEs remove nonpotential, helicity-carrying flux systems from the low corona and into the heliosphere, effectively ridding the erupting volume of magnetic helicity. This allows the magnetic field in the erupting region to relax to its global energetic minimum, the potential state. So, while reconnection without eruption can release some fraction of the corona's free energy, eruption (perhaps with attendant reconnection) can release all of the free energy.

Removing non-potential flux to infinity by ejection has an energetic cost, however, and the coronal field will not spontaneously erupt unless the energetic gain in relaxing to the potential state exceeds the energetic cost of ejecting the non-potential flux. Aly (1984,1991) and Sturrock (1991) argued that sufficient energy to open the field - a presumed requirement to eject a coronal flux system to infinity — cannot be stored in the field by twisting or shearing motions acting on the solar photosphere alone, assuming the field is force free and evolves ideally.

In a series of papers, Linker, Amari, and their collaborators (Linker et al. 2001; Amari et al. 2003a; Amari et al. 2003b), have used MHD simulations to argue that cancellation simultaneously lowers $U_{M}^{(O)}$, the open field energy, while increasing $U_{F}$, although both $U_{M}$ and $U_{M}^{(P)}$ decrease. In these numerical experiments, shear is applied to a model coronal arcade, followed by an imposed electric field on the bottom boundary, which leads to cancellation at the boundary and the formation of a flux rope. Given enough shear and cancellation, the flux rope erupts dynamically.

This paper presents an analytic investigation of the effect of cancellation upon the free magnetic energy in simple models of filament channel fields; its central result is that cancellation in sheared arcades can increase the free magnetic energy in such fields. However, the formalism developed here can be applied more generally, in situations where cancellation plays a minor or negligible role - for instance, in time series of magnetograms, over regions far from any PIL. As it is the free energy that drives flares (for our purposes, reconnective relaxation of the coronal field, with attendant 
particle acceleration, with or without any ejection) and CMEs (eruptive opening of the field in the low corona), this formalism has potential implications for space weather forecasting. Section 2. defines terms and discusses assumptions regarding flux cancellation. Section 3. presents equations to quantify the evolution of free magnetic energy in the coronal field, by separately quantifying the evolution of the actual and potential field energies, and taking their difference. It is then shown that reasonable assumptions about the field configuration in sheared arcades imply that cancellation increases the free magnetic energy in such configurations. Section 4. discusses, in qualitative terms, cancellation and other aspects of field evolution in response to cancellation. Finally, Section 5. briefly outlines the broader applicability and implications of this work.

\section{Definitions of Cancellation}

For this paper, I employ a more mathematical definition of cancellation than that offered above: it occurs when oppositely signed magnetic fluxes threading a surface $S$, in the presence of plasma, come into close proximity and equal amounts of oppositely signed flux "disappear" from $S$ during a time interval $\Delta t$.

I assume that $S$ separates two volumes, $V$ (the corona) and $\tilde{V}$ (the interior), with the plasma $\beta$ (the ratio of gas and magnetic pressures) low in the former and high in the latter. The magnetic Reynolds number is assumed large in both $V$ and $\tilde{V}$, meaning the field evolution is nearly ideal. The surface $S$ is one component of the closed surface $\tilde{S}$ that bounds $V$; the outer surface of $\tilde{S}$ can lie at infinity. The normal field $B_{n}$ might or might not vanish on the rest of the bounding surface, $\tilde{S}-S$. While I will often treat $S$ as the photosphere, it can refer to any atmospheric layer in which cancellation occurs. For instance, Harvey et al. (1999) saw cancellation of the same features in both chromospheric and photospheric magnetograms (though the photospheric cancellation tended to lag behind the chromospheric cancellation), and the following discussion applies to cancellation in either layer. Of course, treating $S$ as a plane is only an approximation, as magnetograms image atmospheric layer of finite thickness, $\delta z$, that is almost certainly not constant in either space or time.

Probably the most straightforward criterion for determining whether cancellation is occurring is a global one: the total amount of unsigned flux through $S$ decreases,

$$
\frac{\partial|\Phi|}{\partial t}=\frac{\partial}{\partial t} \int d S\left|B_{n}\right|<0
$$

This integral constraint, however, is of limited utility in relating flux cancellation to 
the coronal energy budget.

Locally, we know that cancellation of flux in $S$ must occur along a polarity inversion line, a zero contour of $B_{n}$. In the generic case (barring a special symmetry at the PIL), one can adopt a local coordinate system at a point $\mathbf{x}_{0}$ on the PIL, such that $\hat{x} \| \nabla B_{n}\left(\mathbf{x}_{0}\right)$, and $\hat{y} \| \hat{n} \times \nabla B_{n}\left(\mathbf{x}_{0}\right)$. Essentially, $\hat{x}$ points from the negative side of the PIL toward the positive side, and $\hat{y}$ points to the right along the PIL to an observer standing on the positive side of the PIL, as illustrated in Figure 1. By construction, $\partial B_{n} / \partial y$ vanishes in this system. In what follows, this will be the default coordinate system.

Focusing on how magnetic flux is transported to the PIL, I define the ideal flux transport velocity, which is equivalent to Démoulin and Berger's (2003) definition of the pattern velocity of magnetic flux at the photosphere,

$$
\mathbf{u} \equiv \mathbf{v}_{h}-v_{n} \mathbf{B}_{h} / B_{n}
$$

(The subscript $h$ on vector quantities refers to horizontal, by which I mean more precisely the component tangent to $S$.) For Démoulin and Berger, $\mathbf{u}$ is the horizontal velocity at which magnetic features in a magnetogram of $B_{n}$ appear to move when the evolution is ideal; $\mathbf{v}$ is the actual plasma velocity. Non-ideal terms also transport flux, at a rate that can be approximated in Fick's Law form as

$$
B_{n} \mathbf{u}^{(N I)} \equiv-\eta \nabla B_{n}
$$

where $\eta$ is the magnetic diffusivity, equal to $c^{2} / 4 \pi \sigma$, where $\sigma$ is the conductivity. I will assume the diffusive transport is usually negligible, except perhaps near the PIL. The ideal transport of magnetic flux into an subregion $s$ of $S$ is given by

$$
\frac{\partial \Phi_{s}}{\partial t}=-\int d s \nabla_{h} \cdot\left(\mathbf{u} B_{n}\right)=-\oint_{s} d \ell \hat{n} \cdot\left(\mathbf{u} B_{n}\right)
$$

which applies whether the velocity $\mathbf{v}$ is purely vertical, purely horizontal, or has components in both directions. For example, this expression accurately captures the flux transport into $s$ from a moving, tilted magnetic flux tube, whether the tube is rising (or sinking), with $v_{n} \neq 0$ and $\mathbf{v}_{h}=0$, or moving laterally, with $v_{n}=0$ and $\left|\mathbf{v}_{h}\right| \neq 0$. This flux transport velocity describes the evolution of $B_{n}$ in sequences of magnetograms that show cancellation whether reconnection, submergence, or emergence are at work.

Since, evidently, the evolution of $B_{n}$ does not fully constrain the actual plasma flow $\mathbf{v}$, I assume that $\mathbf{v}$ is known in what follows. (Recall that the plasma $\beta$ in $\tilde{V}$, below 
$S$, is assumed high, so the magnetic field within $V$ exerts a negligible force on the plasma in $\tilde{V}$. Hence, the velocity at $S$ is assumed to be kinematically imposed by flows on the upper boundary of $\tilde{V}$, not dynamically determined by the state of the magnetic field in $V$.)

How does one determine if flux is cancelling at the point $\mathbf{x}_{0}$ on the PIL? Fundamentally, the flux transport velocity perpendicular to the PIL must be converging toward the PIL for cancellation to occur. In the local frame, $\partial u_{x} / \partial x<0$ at the PIL is a necessary condition for cancellation to occur. In the archetypal convergence case, $u_{x}=0$ at the PIL, which also makes it a velocity inversion line - a "VIL" for the flux transport velocity perpendicular to the PIL. In the general case, the flux at $\mathbf{x}_{0}$ is being transported with a velocity $\mathbf{u}_{0}$; a Galilean transformation to the comoving frame restores $u_{x}=0$ at $\mathbf{x}_{0}$. (Making $u_{x}=0$ at a different point along the PIL will, in general, require a different transformation.)

A useful local criterion for flux cancellation is that

$$
B_{n} u_{x}<0
$$

is satisfied on both sides of the PIL in the comoving frame. Since only flux transport perpendicular to the PIL instantaneously leads to flux cancellation, flux transport in the $\hat{y}$ direction (parallel to the PIL) does not contribute to cancellation over a small interval $\Delta t$ (to first order), though it can affect the flux of magnetic energy over $\Delta t$.

How does the plasma velocity $\mathbf{v}$ relate to the flux transport velocity $\mathbf{u}$ when cancellation is occurring? If the normal velocity, $v_{n}$, vanishes at the PIL, $v_{x}$ carries normal flux toward the PIL, where it reconnects, as described elsewhere (e.g., Priest and Forbes, 2002). If the evolution is ideal, then the vertical velocity must be nonzero at the PIL, and the disappearance of vertical flux arises by submergence of S-shaped field lines (Chae et al. 2004; Harvey et al. 1999) or emergence of U-shaped field lines (van Driel-Gesztelyi et al. 2000). In ideal case, vertical flux through $S$ smoothly changes to horizontal flux parallel to $S$, which is then advected away from $S$.

\section{Cancellation \& Field Energy}

\subsection{Change in Actual Field Energy}

Rather than calculate the field evolution in $V$ in response to boundary evolution that is consistent with one of the cancellation scenarios above, I will calculate the 
Poynting flux on the boundary, to determine, where possible, changes in the field energy. I assume the electric field $\mathbf{E}$ can be written

$$
\mathbf{E}=-(\mathbf{v} \times \mathbf{B}) / c+\eta(\nabla \times \mathbf{B}) / c,
$$

keeping only the resistive term in Ohm's Law, with $\eta=c^{2} / 4 \pi \sigma$ assumed constant. Then the normal Poynting flux $S_{n}$ of energy into $V$ across $S$ can be written

$$
S_{n}=\frac{c}{4 \pi} \hat{n} \cdot(-(\mathbf{v} \times \mathbf{B}) / c+\eta(\nabla \times \mathbf{B}) / c) \times \mathbf{B} .
$$

If the field is force-free, or the diffusivity is small enough, then the $\eta$ term can be ignored. Since, however, the photosphere is not force-free (Metcalf et al. 1995), and cancellation is often reconnective, I keep it, but express it in terms of $\mathbf{J}$,

$$
S_{n}=\mathbf{B}_{h} \cdot\left(v_{n} \mathbf{B}_{h}-\mathbf{v}_{h} B_{n}\right) / 4 \pi+(\eta / c)\left(\mathbf{J}_{h} \times \mathbf{B}_{h}\right)
$$

For $S_{n}<0$, the magnetic energy in $V$ decreases.

As an aside, since $B_{n}$ is zero at the PIL, the magnetic field near the PIL must be expanded in a Taylor series in the coordinate perpendicular to the PIL,

$$
B_{n} \sim x\left|\nabla B_{n}\right|
$$

to get the Poynting flux density near the PIL. The areal integral over $S_{n}$ in the cancelling region will, in general, be nonzero.

\subsection{Change in Potential Field Energy}

The change in magnetic energy of the potential field cannot be calculated using the Poynting flux, since the induction equation does not govern the evolution between the initial and final potential fields, $\mathbf{B}_{i}^{(P)}$ and $\mathbf{B}_{f}^{(P)}$, in $V$ : the former matches one boundary condition, the latter matches another, but the fields in $V$ do not necessarily conserve topology during the evolution. It is still the case, however, that the induction equation, using the actual tangential magnetic field and the actual plasma flow, governs the normal field's evolution on the boundary. Fortunately, this is all we need to recover the change in energy of the potential field.

Assuming $\mathbf{B}^{(P)}=-\nabla \chi$, one can express the potential magnetic energy as an integral over the surface $\tilde{S}$ that encloses $V$,

$$
U_{m}^{(P)}=\frac{1}{8 \pi} \int d V(\nabla \chi \cdot \nabla \chi)
$$




$$
\begin{aligned}
& =\frac{1}{8 \pi} \oint d \tilde{S} \chi \frac{\partial \chi}{\partial n^{\prime}}-\frac{1}{8 \pi} \int d V \chi \nabla^{2} \chi \\
& =\frac{1}{8 \pi} \oint d \tilde{S} \chi B_{n},
\end{aligned}
$$

where $\nabla \cdot \mathbf{B}^{(P)}=-\nabla^{2} \chi=0$ and $\partial \chi / \partial n^{\prime}=B_{n}$ were used, and the outward and inward normal components were denoted with $n^{\prime}$ and $n$, respectively. Differentiating with respect to time gives

$$
\frac{\partial U_{m}^{(P)}}{\partial t}=\frac{1}{8 \pi} \oint d \tilde{S}\left\{\frac{\partial \chi}{\partial t} B_{n}+\chi \frac{\partial B_{n}}{\partial t}\right\}
$$

Considering the functional dependence of $\chi$ on $B_{n}$ (Jackson 1975) shows that

$$
\frac{1}{8 \pi} \oint d \tilde{S} \frac{\partial \chi}{\partial t} B_{n}=\frac{1}{8 \pi} \oint d \tilde{S} \oint d \tilde{S}^{\prime} \frac{\partial B_{n}\left(\mathbf{x}^{\prime}\right)}{\partial t} B_{n}(\mathbf{x}) \frac{1}{\left|\mathbf{x}-\mathbf{x}^{\prime}\right|}
$$

Since the integrations commute,

$$
\oint d \tilde{S} \frac{\partial \chi}{\partial t} B_{n}=\oint d \tilde{S} \chi \frac{\partial B_{n}}{\partial t}
$$

meaning

$$
\frac{\partial U_{m}^{(P)}}{\partial t}=\frac{1}{4 \pi} \oint d \tilde{S} \chi \frac{\partial B_{n}}{\partial t}=\frac{1}{4 \pi} \int d S \chi \frac{\partial B_{n}}{\partial t} .
$$

The final step keeps only the surface term corresponding to magnetic field changes on $S$, the bottom portion of $\tilde{S}$, on the assumption that magnetic field changes on the other components of $\tilde{S}$ are negligible. Referring to the induction equation (with $\sigma$ constant in space, and the actual $\mathbf{B}_{h}$ ),

$$
\frac{\partial B_{n}}{\partial t}=\nabla_{h} \cdot\left(v_{n} \mathbf{B}_{h}-\mathbf{v}_{h} B_{n}\right)-(c / \sigma)\left(\nabla_{h} \times \mathbf{J}_{h}\right),
$$

one sees that

$$
\begin{aligned}
\frac{\partial U_{m}^{(P)}}{\partial t} & =\frac{1}{4 \pi} \int d S \chi \nabla_{h} \cdot\left(v_{n} \mathbf{B}_{h}-\mathbf{v}_{h} B_{n}\right)-\frac{c}{4 \pi \sigma} \int d S \chi\left(\nabla_{h} \times \mathbf{J}_{h}\right) \\
& =-\frac{1}{4 \pi} \int d S \nabla_{h} \chi \cdot\left(v_{n} \mathbf{B}_{h}-\mathbf{v}_{h} B_{n}\right)+\frac{c}{4 \pi \sigma} \int d S\left(\nabla_{h} \chi \times \mathbf{J}_{h}\right) \\
& +\frac{1}{4 \pi} \oint_{S} d \ell \chi \hat{n}_{S} \cdot\left(v_{n} \mathbf{B}_{h}-\mathbf{v}_{h} B_{n}\right)-\frac{c}{4 \pi \sigma} \oint_{S} d \hat{\ell} \cdot\left(\chi \mathbf{J}_{h}\right) \\
& =\frac{1}{4 \pi} \int d S \mathbf{B}_{h}^{(P)} \cdot\left(v_{n} \mathbf{B}_{h}-\mathbf{v}_{h} B_{n}\right)-\frac{c}{4 \pi \sigma} \int d S\left(\mathbf{B}_{h}^{(P)} \times \mathbf{J}_{h}\right),
\end{aligned}
$$


where, assuming that the field is sufficiently localized on $S$, one can neglect the line integrals in the partial integrations on the boundaries of $S$. One can now express the change in the potential field's magnetic energy as a Poynting-like energy flux density that depends upon both the actual and potential fields, $\mathbf{B}$ and $\mathbf{B}^{(P)}$, and the plasma velocity $\mathbf{v}$

$$
S_{n}^{(P)}=\mathbf{B}_{h}^{(P)} \cdot\left(v_{n} \mathbf{B}_{h}-\mathbf{v}_{h} B_{n}\right) / 4 \pi+(\eta / c)\left(\mathbf{J}_{h} \times \mathbf{B}_{h}^{(P)}\right) .
$$

If one included any additional term(s) in the generalized Ohm's Law in equation (6), corresponding terms would appear in both equations (8) and (21), with $\mathbf{B}_{h}$ in the former replaced by $\mathbf{B}_{h}^{(P)}$ in the latter.

\subsection{Change in Free Energy}

The flux of free magnetic energy in the corona, $S_{n}^{(F)}$, is given by the difference between $S_{n}$ and $S_{n}^{(P)}$,

$$
\begin{aligned}
S_{n}^{(F)} & =\left(S_{n}-S_{n}^{(P)}\right) / 4 \pi \\
& =\left(\mathbf{B}_{h}-\mathbf{B}_{h}^{(P)}\right) \cdot\left(v_{n} \mathbf{B}_{h}-\mathbf{v}_{h} B_{n}\right) / 4 \pi \\
& +(\eta / c)\left(\mathbf{J}_{h} \times\left(\mathbf{B}_{h}-\mathbf{B}_{h}^{(P)}\right)\right) \cdot \hat{n}
\end{aligned}
$$

The goal of this paper is to determining the sign of $S_{n}^{(F)}$ in equation (23) in the specific case of cancellation in sheared arcade models of filament channel fields. The formalism in equation (23) can, however, be applied more generally, a point I digress to consider here.

According to all the observations of cancellation cited above (perhaps excluding that of van Driel-Gesztelyi et al. [2000]), cancellation occurs only intermittently, between small, isolated patches of flux. Consequently, the magnetic field in most of the area covered by a magnetograph's field of view evolves essentially ideally, at least over the time scales of less than a day - the typical time scale of flare and CME energization, assuming this is related to flare and CME recurrence rates (Wheatland and Litvinenko 2002; Wheatland 2003). Hence, the ideal form of equation (23),

$$
S_{n}^{(F)}=\left(\left(\mathbf{B}_{h}-\mathbf{B}_{h}^{(P)}\right) \cdot\left(v_{n} \mathbf{B}_{h}-\mathbf{v}_{h} B_{n}\right) / 4 \pi,\right.
$$

should approximate the flux of free energy across the surface imaged by a magnetogram.

Applied to time series of vector magnetograms, and combined with techniques for deriving plasma velocities, such as ILCT (Welsch et al. 2004), MEF (Longcope 2004), 
and others (Kusano et al. 2002; Kusano 2005; Schuck 2005), equation (23) allows calculation of the change in free magnetic energy in the corona. Démoulin and Berger (2003) have argued that flows $\mathbf{u}^{(L C T)}$ determined by local correlation tracking (LCT), when multiplied by $B_{n}$, are formally equivalent to the quantity $-\left(v_{n} \mathbf{B}_{h}-\mathbf{v}_{h} B_{n}\right)$ from equation (23), although Schuck (2005) has argued that this equivalence is only approximate. Non-linear force-free field inversion techniques (Schrijver et al. 2005) could be used to determine an initial estimate of the free magnetic energy. Hence, the budget of free magnetic energy in the corona can be estimated, a point revisited in the concluding section.

I note that, in principle, the non-ideal term in equation (23) could also be included in such an analysis, with either a suitable data set, or simplifying assumptions. This term contains $J_{x}$ and $J_{y}$, and therefore involves vertical derivatives of the magnetic field's horizontal components. These two quantities are not currently available with most vector magnetogram observations, which invert Stokes' profiles over a single, relatively thin, atmospheric layer (but see Metcalf et al. [1995]). Recent modifications to the University of Hawaii's imaging vector magnetograph (IVM) should allow nearly simultaneous acquisition of chromospheric and photospheric polarimetric data, suitable for producing nearly simultaneous vector magnetograms of both atmospheric layers (Mickey and Metcalf 2003), thereby determining the horizontal current $\mathbf{J}_{h}$. A quantitative approximation to $\mathbf{J}_{h}$ could also be made from a magnetogram at a single height by adopting a surface diffusion model, in which the vertical derivatives of $B_{x}$ and $B_{y}$ are neglected in computing $J_{y}$ and $J_{x}$ (see, e.g., van Ballegooijen et al. 2000). Chae et al. (2002) derived an estimate of $\eta$ from observations of a putative reconnection event near the temperature minimum, between the photosphere and chromosphere, the expected site of magnetic reconnection (Litvinenko 1999). Hence, the necessary pieces are in place to use equation (23) to observationally estimate the free energy flux into the corona, even when the field evolution is not ideal.

\subsection{Cancellation within Sheared Arcades}

I return now to the main theme of analyzing the free energy flux from cancellation in sheared arcades. Cancelling flows are of the form $\mathbf{v}=\left(v_{x}, 0, v_{n}\right)^{T}$, so the rate of change of free energy becomes

$$
\begin{aligned}
S_{n}^{(F)} & =\left(v_{n} B_{x}-B_{n} v_{x}\right)\left(B_{x}-B_{x}^{(P)}\right) / 4 \pi+v_{n} B_{y}\left(B_{y}-B_{y}^{(P)}\right) / 4 \pi \\
& +(\eta / c)\left[J_{x}\left(B_{y}-B_{y}^{(P)}\right)-J_{y}\left(B_{x}-B_{x}^{(P)}\right)\right] .
\end{aligned}
$$


By examining the terms in equation (25) in detail, one can determine the sign of $S_{n}^{(F)}$ during cancellation. To make progress, I make the following assumptions about $\mathbf{B}$ and $\mathbf{B}^{(P)}$ in filament channels and sheared arcades,

$$
\begin{aligned}
B_{x}^{(P)} & <0 \\
\left|B_{y}^{(P)}\right| & \ll\left|B_{y}\right| .
\end{aligned}
$$

Assuming the criterion for flux cancellation, equation (5), is satisfied, $-B_{n} u_{x}=$ $\left(v_{n} B_{x}-v_{x} B_{n}\right)>0$ near the neutral line.

Next, I consider the sign of difference between the actual and potential horizontal fields perpendicular to the PIL, $\left(B_{x}-B_{x}^{(P)}\right)$. If field lines arch over the PIL, from $B_{n}>0$ to $B_{n}<0$ across the PIL, then $B_{x}$ points in the conventional sense, and $B_{x}<0$ in the local frame. If, however, $B_{x}$ points in the "inverse" direction, such that $B_{x}>0$ at the PIL, then field lines are dipped over the PIL. Dipped field lines over the PIL can occur in various configurations, notably at a bald patch in a potential field (Titov et al. 1993), or when the lowest field line in a horizontal flux rope osculates $S$ in a current-carrying configuration. Figure 2 shows two simple configurations in which the sign of $B_{x}$ varies, based upon the field's topology above the PIL. Ignoring potential field configurations with dipped field lines over the PIL, I assume that field lines in $\mathbf{B}^{(P)}$ generally arch over the PIL conventionally, consistent with equation (26).

If the actual field lines dip over the PIL, $B_{x}>0$, as with a flux rope, and field lines of $B_{x}^{(P)}$ arch over the PIL, then the difference $\left(B_{x}-B_{x}^{(P)}\right)$ in equation (25) is positive.

In a bipolar arcade where both the actual and potential field lines arch over the PIL in the conventional sense, both $B_{x}<0$ and $B_{x}^{(p)}<0$. In a sheared bipolar arcade, the actual field has a component along the PIL, $B_{y} \neq 0$, while the potential field is essentially perpendicular to the PIL, $B_{y} \simeq 0$. Klimchuk (1990) analyzed such configurations and found that increasing shear — essentially, increasing $\left|B_{y}\right|$ - invariably increases the height at which field lines of $\mathbf{B}$ cross the PIL. Since the potential state has minimal shear, this inflation of the arcade has the consequence that the average flux density perpendicular to the PIL, $B_{x}$, decreases compared to the potential state, so

$$
\left|B_{x}^{(P)}\right|>\left|B_{x}\right|
$$

obtains near the PIL, meaning $\left(B_{x}-B_{x}^{(P)}\right)$ in equation (25) is, again, positive.

It is perhaps not obvious that $\left|B_{x}^{(P)}\right|>\left|B_{x}\right|$, so I illustrate this point with a well- 
known, Cartesian, periodic model of a force-free, sheared arcade, where

$$
\begin{aligned}
& B_{x}(x, y, z)=-\sqrt{1-\left(\alpha / k_{x}\right)^{2}} B_{0} \sin \left(k_{x} x\right) \exp \left(-k_{z} z\right) \\
& B_{y}(x, y, z)=-\left(\alpha / k_{x}\right) B_{0} \sin \left(k_{x} x\right) \exp \left(-k_{z} z\right) \\
& B_{z}(x, y, z)=-B_{0} \cos \left(k_{x} x\right) \exp \left(-k_{z} z\right) .
\end{aligned}
$$

The potential field is given by setting $\alpha=0$; field lines lie in constant- $y$ planes, and arch over the PIL at $x=\pi / 2 k_{x}$. Choosing $\alpha \neq 0$ implies $B_{y} \neq 0$, and gives a simple model of a non-potential, force-free sheared arcade in which field lines lie in planes tilted with respect to constant- $y$ planes by an angle $\theta=\tan ^{-1}\left(\alpha / \sqrt{k_{x}^{2}-\alpha^{2}}\right)$. From equation (29), it can easily be seen that $\left|B_{x}^{(P)}\right|>\left|B_{x}\right|$ for $\alpha \neq 0$, and Figure 3 demonstrates this graphically by displaying the inflated flux surfaces, and resulting decreased flux density $B_{x}$ across the PIL, in the $\alpha \neq 0$ case.

When the field near the PIL exhibits an X-point topology, $B_{x} \sim 0$ near the PIL, so the direction of $B_{x}$ at $S$ is poorly defined. Since, by assumption, the potential field does have a well defined direction, the difference $\left(B_{x}-B_{x}^{(P)}\right)$ also has a well defined sign.

In any case, then, the $\left(B_{x}-B_{x}^{(P)}\right)$ term in equation $(25)$ is positive, whether the horizontal field perpendicular to the PIL points in the conventional or inverse sense, and $\left(B_{x}-B_{x}^{(P)}\right)\left(v_{n} B_{x}-v_{x} B_{n}\right)>0$ for cancellation in both cases. This result depends upon the assumption in equation (26); if the potential field has a bald patch at the PIL, this result does not necessarily follow.

Equation (27) implies the second term in equation (25), $v_{n} B_{y}\left(B_{y}-B_{y}^{(P)}\right)$, has the same sign as $v_{n}$.

Finally, I analyze the sign of $S_{n}^{(F, N I)}$, the non-ideal contribution to the vertical flux of free energy,

$$
S_{n}^{(F, N I)}=(\eta / c)\left[J_{x}\left(B_{y}-B_{y}^{(P)}\right)-J_{y}\left(B_{x}-B_{x}^{(P)}\right)\right]
$$

in the case of reconnective cancellation, when a thin current sheet exists along the interface between the cancelling normal-flux features. The lack of strong magnetic field across the sheet in the reconnecting region implies $\left|B_{x}\right| \simeq 0$, while the close proximity of the cancelling flux implies $\left|B_{x}^{(P)}\right|$ is relatively large, with $B_{x}^{(P)}<0$. This fact and the assumption in equation (27) imply

$$
S_{n}^{(F, N I)} \simeq(\eta / c)\left(J_{x} B_{y}+J_{y} B_{x}^{(P)}\right)
$$

The high current density in the sheet implies $\left|J_{y}\right| \gg\left|J_{x}\right|$, with $J_{y}<0$, while the magnitude of the guide field, $B_{y}$, if any, would be of the same order as $\left|B_{x}^{(P)}\right|$. Consequently, the sign of $S_{n}^{(F, N I)}$ is determined by the sign of the product $J_{y} B_{x}^{(P)}$, which is 
positive. Evidently, in this model of cancellation in a sheared arcade, the dissipative removal of normal flux drops the energy of the potential field faster than it drops the energy of the actual field. This result can be explained by noting that dissipation removes strong field — that which straddles the PIL — from the potential system, while reconnection can merely link distant flux systems in a non-potential system.

For reconnective cancellation $\left(v_{n}=0\right)$ in a sheared arcade, then, $S_{n}^{(F)}>0$. For cancellation via emergence, the flux of free energy into the corona is greater than or equal to zero. For cancellation via submergence, the situation is more complicated; this point is discussed in more detail below. When the actual field's topology is inverse, but the potential field's is conventional, the difference $\left(B_{x}-B_{x}^{(P)}\right)$ is greater than if both fields have conventional topology. Consequently, the free energy flux from cancellation is larger in the inverse $B_{x} /$ conventional $B_{x}^{(P)}$ case, which suggests that cancellation should have a greater effect on the free energy in such configurations.

\section{Discussion}

\subsection{Cancellation and $\partial B_{y} / \partial t$}

An essential difference between ideal cancellation and reconnective cancellation is the transport of flux, $\Phi_{y}$, that threads the $x-z$ plane (or runs parallel to the PIL) in the local frame. With either ideal submergence of $\Omega$-shaped field lines or ideal emergence of U-shaped field lines, $\Phi_{y}$ is merely advected with the flow. With reconnective cancellation, however, both diffusion and material flow transport $\Phi_{y}$ away from the reconnection line, in $S$.

How does $B_{y}$ evolve above and below $S$ as reconnective cancellation proceeds? Though

$\Phi_{y}$ is conserved during three-dimensional reconnection, $B_{y}$ is not: once initially parallel flux has advected through the diffusion region, it can rotate as it relaxes to accommodate its new topology. For reconnective cancellation above the photosphere in a sheared arcade, footpoints of reconnected field lines below the reconnection site straddle the PIL, and so are closer in $y$ than footpoints of reconnected field lines above reconnection site. Consequently, reconnected flux below the reconnection region tends to run in the $\hat{x}$ direction, while flux above it runs in the $\hat{y}$ direction, as seen in the numerical simulations of Welsch et al. (2005). Also, even with $\left|\partial B_{y} / \partial z\right| \neq 0, B_{y}$ does not undergo a field reversal across the reconnection site like $B_{n}$ does. This means that diffusivity leads to reconnection of $B_{n}$ at the PIL, but does not necessarily imply that $\left|B_{y}\right|$ diffuses downward; the gradient in the $y$-analog of equation (3) is much smaller 
than $\nabla B_{n}$. Van Ballegooijen and Martens (1989) argued that this decoupling of $B_{y}$ and the reconnecting field components was necessary for the subsequent cancellation of the reconnected loops via submergence.

\subsection{Convergence, Reconnection, and Submergence}

Convergence of oppositely signed magnetic flux distributions at the photosphere increases the free magnetic energy in the coronal field. By analogy with charges in electrostatics, the motion of opposing fluxes toward each other lowers the potential field's energy. In the actual field, however, the presence of shear and the constraint of conserving topology mean that the opposing fluxes are not magnetically linked in $V$, so the motion of the opposing fluxes toward each other increases the actual field's energy: a current sheet develops between unconnected flux systems, and such currents store free magnetic energy.

If the flux distributions become close enough, magnetic reconnection, in a layer $S_{+}$ above the photosphere, can create new field linkages between the two. While the reconnection will reduce the magnetic energy in the system, it will not destroy all the magnetic helicity present (Berger 1984), so the post-reconnection field will not be potential. The analysis below equation (33) suggests that the dissipation process itself leads to an increase in the free energy, although both the actual and potential energies drop.

If the flux distributions subsequently cancel at the photosphere, the cancellation corresponds to the submergence of post-reconnection $\Omega$ loops. This scenario is consistent with the observations reported by Harvey et al. (1999) for quiet Sun magnetic fluxes, and was simulated by Welsch et al. (2005) to study prominence formation.

Is the change in free energy in the corona in this picture still positive, or does the submergence remove free energy? Van Ballegooijen and Martens (1989) argued that the photosphere would act as a barrier to the submergence of all but strongly arched loops; magnetic tension could only overcome magnetic buoyancy near the photosphere for loops with closely spaced footpoints. Hence, the submerging flux will be essentially potential: the footpoints of the submerging loop must closely straddle the PIL, and be close together in $y$, meaning $B_{y} \simeq B_{y}^{(P)} \simeq 0$ in the submerging field, and $\left(B_{x}-B_{x}^{(P)}\right) \simeq$ 0 , so $S_{n}^{(F)} \simeq 0$. I speculate that, to the extent that the post-reconnection fields are potential, their submergence does not remove more free magnetic energy than was added to the coronal field by: 1) the converging motions prior to the reconnection, 
and 2) the dissipation process. Essentially, some free energy would remain "trapped" in the corona, consistent with the simulations of Welsch et al. (2005).

\subsection{Cancellation and Magnetic Helicity}

In addition to considering the flux of magnetic energy during cancellation, I also briefly consider the evolution of magnetic helicity in $V$ during the cancellation process, by considering the flux of helicity across $S$ during the cancellation process. Obviously, the emergence of a flux rope, which by definition would contain helicity, would lead to a flux of helicity into the corona.

The case of reconnective cancellation $\left(v_{n}=0\right.$ at $\left.S\right)$ followed by submergence is more interesting. Berger and Field (1984) derived an expression for the flux of gaugeinvariant relative helicity across the surface $S$ that bounds the volume $V$,

$$
\frac{d \mathcal{H}}{d t}=2 \int d S\left[\left(\mathbf{A}^{(P)} \cdot \mathbf{B}_{\mathrm{h}}\right) v_{n}-\left(\mathbf{A}^{(P)} \cdot \mathbf{v}_{\mathrm{h}}\right) B_{n}\right],
$$

where $\mathbf{A}^{(P)}$, the vector potential for $\mathbf{B}^{(P)}$. After Berger (1999), assuming

$$
\begin{array}{r}
\left(\nabla \times \mathbf{A}^{(P)}\right) \cdot \hat{n}=B_{n} \\
\left(\nabla \cdot \mathbf{A}^{(P)}\right)=0 \\
\left.\mathbf{A}^{(P)} \cdot \hat{n}\right|_{S}=0
\end{array}
$$

uniquely specifies the vector potential's $i$ th component,

$$
A_{i}^{(P)}=\int d S \frac{B_{n}\left(x^{\prime}, y^{\prime}\right)\left(\hat{n} \times\left(x_{j}-x_{j}^{\prime}\right) \hat{x}_{j}\right) \varepsilon_{i j n}}{\left|\left(x_{i}-x_{i}^{\prime}\right)+\left(x_{j}-x_{j}^{\prime}\right)\right|^{2}} .
$$

For a system invariant in $y$, the numerator in the integration for $A_{x}^{(P)}$ is odd in $y$, so $A_{x}^{(P)}=0$. For an example of the vector potential in a typical bipolar active region, see Chae (2001). Hence, there is no helicity flux for flows perpendicular to the PIL, $\mathbf{v}_{h} \| \hat{x}$. To the extent that a filament channel can be approximated as invariant along the PIL (or exhibits some other form of symmetry), the helicity flux from reconnective cancellation approximately vanishes.

Qualitatively, footpoints of field lines that cancel do not wind about each other; such winding is necessary to generate a flux of helicity. This essentially local analysis, however, ignores the fact that, as the converging footpoints approach each other, they could be winding about other magnetic flux distributions away from the cancellation 
site, thereby generating mutual helicity (Berger 1999) with the distant magnetic flux distributions. (By virtue of their opposing velocities and polarities, their contributions to this mutual helicity would reinforce each other.)

If the reconnection takes place above the photosphere, does the submergence of postreconnection, inverse- $\mathrm{U}$ loops, as described above, remove a significant amount of helicity from the system? As noted above, the footpoints of the submerging, postreconnection loops closely straddle the PIL, meaning the loops are short. Conse-

quently, large values of twist per unit length would be required for the submerging flux system to remove a significant fraction of the coronal helicity.

The removal of flux from the corona by the cancellation without removal of significant helicity implies that whatever helicity was present prior to the cancellation must now be distributed over less flux. If the only flux that reconnects is that near the PIL, then the helicity is only distributed within the flux near the PIL (Antiochos et al. 2002). Qualitatively, then, cancellation has the property of concentrating helicity near PILs, not diffusing it, implying that cancellation leads to variable- $\alpha$ fields - not the constant- $\alpha$ state predicted by Taylor's (1985) hypothesis. It has been noted before that the coronal field does not appear to diffuse non-potentiality, in the form of helicity (e.g., Antiochos 1999), a fact that is hard to explain in terms of flaring and reconnection in the corona, which presumably act to distribute variations in current density more evenly (Nandy et al. 2003).

\section{Implications \& Conclusions}

I derived an expression, equation (23), for the flux of free magnetic energy into the corona. For the special case of sheared-arcade filament channels, analysis of the ideal terms shows that the approach of cancelling features toward the PIL increases the coronal free energy, while the contribution from non-ideal terms during reconnective cancellation also increases the coronal free magnetic energy. This result is true whether the topology in filament channels is that of a flux rope or sheared, arched field lines. I speculate that, when cancellation occurs via reconnection above the photosphere, followed by the submergence of nearly potential, post-reconnection loops, the submergence does not remove as much free energy as was added during the approach and reconnection phases.

To the extent that cancellation processes do add free energy to the coronal magnetic field, observations of flux cancellation would suggest that the energy available for to 
drive flares and/or CMEs is increasing, meaning such events would be more likely. The formalism developed here for application to the particular case of flux cancellation has much wider applicability. Equation (21) is a Poynting-like energy flux density for the potential magnetic field in terms of plasma velocities, useful for determining which flows lead to changes in the energy of the potential field, information that is unavailable when the integral expressions in equations (10) or (12) are used to determine the potential field energy at the endpoints of a short time interval $\Delta t$. Similarly, equation (23) enables analysis of which flows lead to significant changes in free energy; again, this information is unavailable from integral expressions for the actual and potential magnetic energies (e.g., Metcalf 2002).

Modellers can use the energy flux densities in equations (21) and (23) to analyze fluxes in potential field energy and free energy in MHD simluations of coronal evolution. Observers can also use these expressions, in combination with techniques for deriving plasma velocities from time series of vector magnetograms, such as LCT (Démoulin and Berger 2003; Welsch et al. 2004), ILCT (Welsch et al. 2004), MEF (Longcope 2004) and others (Kusano et al. 2002; Kusano 2005; Schuck 2005), to derive the flux of magnetic free energy into the corona. This application would require determination of the horizontal components of the potential magnetic field, $\mathbf{B}_{h}^{(P)}$, at each time step, from the actual $B_{n}$. Whether for simulations or magnetograms, these components can be determined in straightforward ways, by incorporating the normal field into an appropriate global field (for instance, a synoptic magnetogram for solar data), and applying Fourier or Green's function techniques.

I note also that the integral expression used by Metcalf (2002) requires the use of chromospheric vector magnetograms, because the field must be force-free on the boundary (Metcalf et al. 1995) to evaluate the magnetic energy in the coronal volume in terms of a surface integral. The free energy flux formalism developed here, however, can be applied to photospheric magnetograms, which means many more data sets are available for such analysis. Further, with the advent of round-the-clock, high resolution vector magnetograms from SDO-HMI, this free energy flux formalism holds promise as a forecast tool for space weather applications.

Estimates of free energy flux from observations could be used to validate competing models of CME initiation, e.g., the 'driven CME' model of Chen (1996), in which CMEs are driven directly by emergence of free magnetic energy through the photosphere, versus the breakout (Antiochos et al. 1999; MacNeice et al. 2004) and flux cancellation (Linker et al. 2001; Amari et al. 2003a; Amari et al. 2003b) models, in which CMEs occur via loss of equilibrium in the corona, without direct photospheric 
driving of the ejection. Knowledge of the sites of free energy injection, perhaps coupled with a topological model of the overlying coronal field, could also improve our understanding of processes by which free energy builds up in the corona, e.g. by shearing along PILs (Antiochos et al. 1999; MacNeice et al. 2004), or by rotation of sunspots (Brown et al. 2003).

I thank Spiro Antiochos, George Fisher, Piet Martens, and Gunnar Hornig for their insightful comments regarding the evolution of the coronal field and flux cancellation as I was developing the ideas presented here, members of SSL's Friday Solar Lunch group for reviewing this manuscript prior to its submission, and the anonymous referee whose careful review led to subtantial improvements in the manuscript. I also appreciate the kind support of AFOSR's MURI and NASA's SEC Theory programs.

\section{REFERENCES}

Aly, J. J. 1984, ApJ 283, 349.

Aly, J. J. 1991, ApJ375, L61.

Amari, T., Luciani, J. F., Aly, J. J., Mikic, Z., and Linker, J. 2003a, ApJ 585, 1073.

Amari, T., Luciani, J. F., Aly, J. J., Mikic, Z., and Linker, J. 2003b, ApJ595, 1231.

Antiochos, S. K. and Devore, C. R. 1999, The Role of Helicity in Magnetic Reconnection: 3D Numerical Simulations, in Magnetic Helicity in Space and Laboratory Plasmas, AGU Monograph, volume 111, p. 187, Washington, AGU.

Antiochos, S. K., DeVore, C. R., and Klimchuk, J. A. 1999, ApJ 510, 485.

Antiochos, S. K., Karpen, J. T., and DeVore, C. R. 2002, ApJ 575, 578.

Aulanier, G. and Démoulin, P. 2003, A\&A402, 769.

Aulanier, G., Srivastava, N., and Martin, S. F. 2000, ApJ543, 447.

Berger, M. 1999, Magnetic Helicity in Space Physics, in Magnetic Helicity in Space and Laboratory Plasmas, pp. 1-9, AGU.

Berger, M. A. 1984, Geophys. Astrophys. Fluid Dynamics 30, 79.

Berger, M. A. and Field, G. B. 1984, JFM 147, 133. 
Bommier, V. and Leroy, J. L. 1998, Global Pattern of the Magnetic Field Vectors Above Neutral Lines from 1974 to 1982: Pic-du-Midi Observations of Prominences, in ASP Conf. Ser. 150: IAU Colloq. 167: New Perspectives on Solar Prominences, pp. 434-+.

Brown, D. S., Nightingale, R. W., Alexander, D., Schrijver, C. J., Metcalf, T. R., Shine, R. A., Title, A. M., and Wolfson, C. J. 2003, Sol. Phys.216, 79.

Chae, J. 2001, ApJ 560, L95.

Chae, J., Moon, Y., and Park, S. 2003, J. Korean Astron. Soc. 36, 13.

Chae, J., Moon, Y., and Pevtsov, A. A. 2004, ApJ 602, L65.

Chen, J. 1996, J. Geophys. Res.101(10), 27499.

Démoulin, P. and Berger, M. A. 2003, Sol. Phys. 215, 203.

Démoulin, P. 1998, Magnetic Fields in Filaments (Review), in ASP Conf. Ser. 150: IAU Colloq. 167: New Perspectives on Solar Prominences, pp. 78-+.

Fan, Y. 2001, ApJ554, L111.

Forbes, T. G. 2000, JGR 105, 23153.

Gosling, J. T. 1993, JGR 98(A11), 18,937.

Harvey, K. L., Jones, H. P., Schrijver, C. J., and Penn, M. J. 1999, Solar Phys. 190, 35.

Jackson, J. D. 1975, Classical Electrodynamics, Wiley, New York.

Klimchuk, J. A. 1990, ApJ354, 745.

Kusano, K. 2005, in Sakurai, T. and Skii, T., editors, The Solar-B Mission and the Forefront of Solar Physics, volume ASP Conf. Ser. 325, p. in press, San Francisco, ASP.

Kusano, K., Maeshiro, T., Yokoyama, T., and Sakurai, T. 2002, ApJ 577, 501.

Linker, J. A., Lionello, R., Mikić, Z., and Amari, T. 2001, JGR 106, 25165.

Litvinenko, Y. E. 1999, ApJ 515, 435. 
Livi, S. H. B., Wang, J., and Martin, S. F. 1985, Australian Journal of Physics 38, 855.

Longcope, D. W. 2004, ApJ 612.

Low, B. C. 2002, Magnetic coupling between the corona and the solar dynamo, in ESA SP-505: SOLMAG 2002. Proceedings of the Magnetic Coupling of the Solar Atmosphere Euroconference, pp. 35-+.

Low, B. C. and Hundhausen, J. R. 1995, ApJ 443, 818.

MacNeice, P., Antiochos, S. K., Phillips, A., Spicer, D. S., DeVore, C. R., and Olson, K. 2004, ApJ614, 1028.

Magara, T., Antiochos, S. K., Devore, C. R., and Linton, M. G. 2005, AGU Spring Meeting Abstracts, $\mathrm{C} 1+$.

Martens, P. C. and Zwaan, C. 2001, ApJ 558, 872.

Martin, S. F. 1998, Sol. Phys. 182, 107.

Metcalf, T. R., Jiao, L., McClymont, A. N., Canfield, R. C., and Uitenbroek, H. 1995, ApJ 439, 474.

Metcalf, T. R., Mickey, D. L., Labonte, B. J., and Ryder, L. A. 2002, The Magnetic Free Energy and a CME in Active Region 8299, in Multi-Wavelength Observations of Coronal Structure and Dynamics, pp. 249-+.

Mickey, D. and Metcalf, T. R. 2003, Private Communication , N/A.

Nandy, D., Hahn, M., Canfield, R. C., and Longcope, D. W. 2003, ApJ597, L73.

Rust, D. M. and Kumar, A. 1994, Solar Phys. 155, 69.

Schrijver, C. J., Derosa, M. L., and Metcalf, T. 2005, AGU Spring Meeting Abstracts , A $5+$.

Schuck, P. 2005, AGU Spring Meeting Abstracts .

Sturrock, P. A. 1991, ApJ380, 655.

Sudol, J. J., Harvey, J. W., and Howe, R. 2004, American Astronomical Society Meeting Abstracts 204, .

Taylor, J. B. 1986, Rev. Mod. Phys. 58(3), 741. 
Titov, V. S., Priest, E. R., and Demoulin, P. 1993, A\&A276, 564.

van Ballegooijen, A. A. and Martens, P. C. H. 1989, ApJ 343, 971.

van Ballegooijen, A. A., Priest, E. R., and Mackay, D. H. 2000, ApJ539, 983.

van Driel-Gesztelyi, L., Malherbe, J.-M., and Démoulin, P. 2000, A\&A364, 845.

Welsch, B. T., DeVore, C., and Antiochos, S. 2005, ApJ (in press).

Welsch, B. T., Fisher, G., and Abbett, W. 2004, ApJ 610, 1148.

Wheatland, M. S. 2003, Sol. Phys.214, 361.

Wheatland, M. S. and Litvinenko, Y. E. 2002, Sol. Phys.211, 255.

Yurchyshyn, V. B. and Wang, H. 2001, Sol. Phys.202, 309.

Zwaan, C. 1987, ARA\&A 25, 83. 
Fig. 1 - Grayscale indicates $B_{n}$ in this schematic representation of the local coordinate system at a point $\mathbf{x}_{0}$ on the PIL (dashed line). Essentially, $\hat{x} \| \nabla B_{n}$ points from the PIL's negative side toward the positive side, and $\hat{y}$ points to the right along the PIL to an observer standing on the positive side of the PIL.

Fig. 2 - a) The normal magnetic field $B_{n}$ near the PIL in a plane parallel to and two units away from a line current. b) Selected field lines if the current source is below the plane. c) Selected field lines arising from the same boundary condition, but with the current source above the plane.

Fig. 3 - In a simple sheared arcade model, both the actual field $B_{x}$ (dashed field lines are projections onto the plane of the page) and $B_{x}^{(P)}$ (solid field lines) are both negative. The grayscale image of $\left(B_{x}-B_{x}^{(P)}\right)$, in which white is positive, shows that $B_{x}$ is less negative than $B_{x}^{(P)}$ below the dotted line, since the shear inflates the field vertically (Klimchuk 1990). 


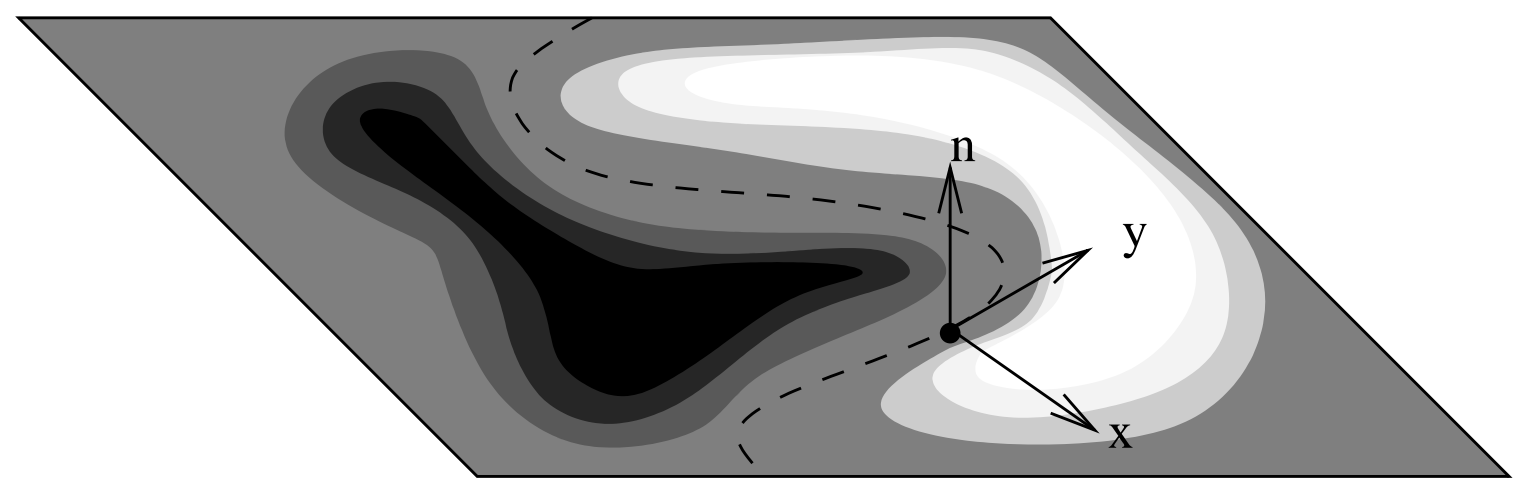

Fig. 1.- 
a)

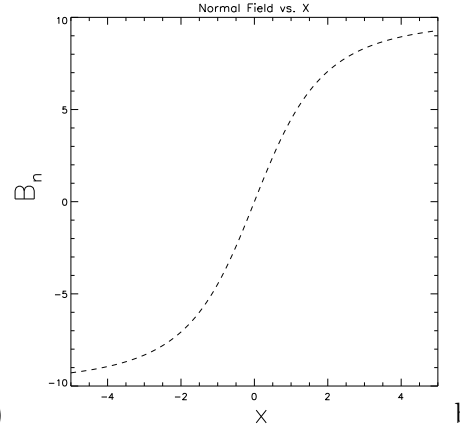

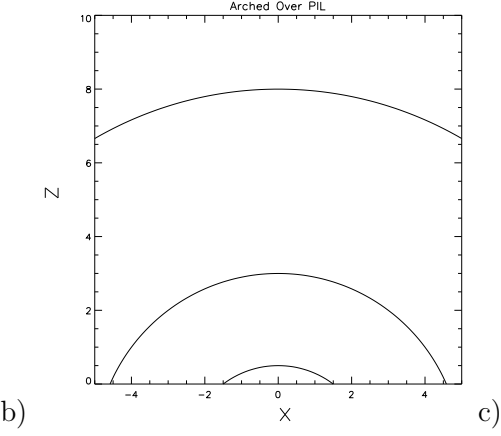

c)

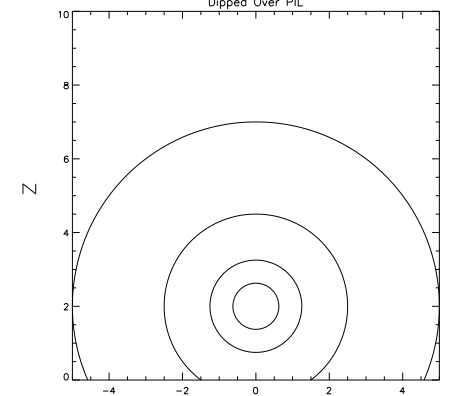

Fig. 2.- 


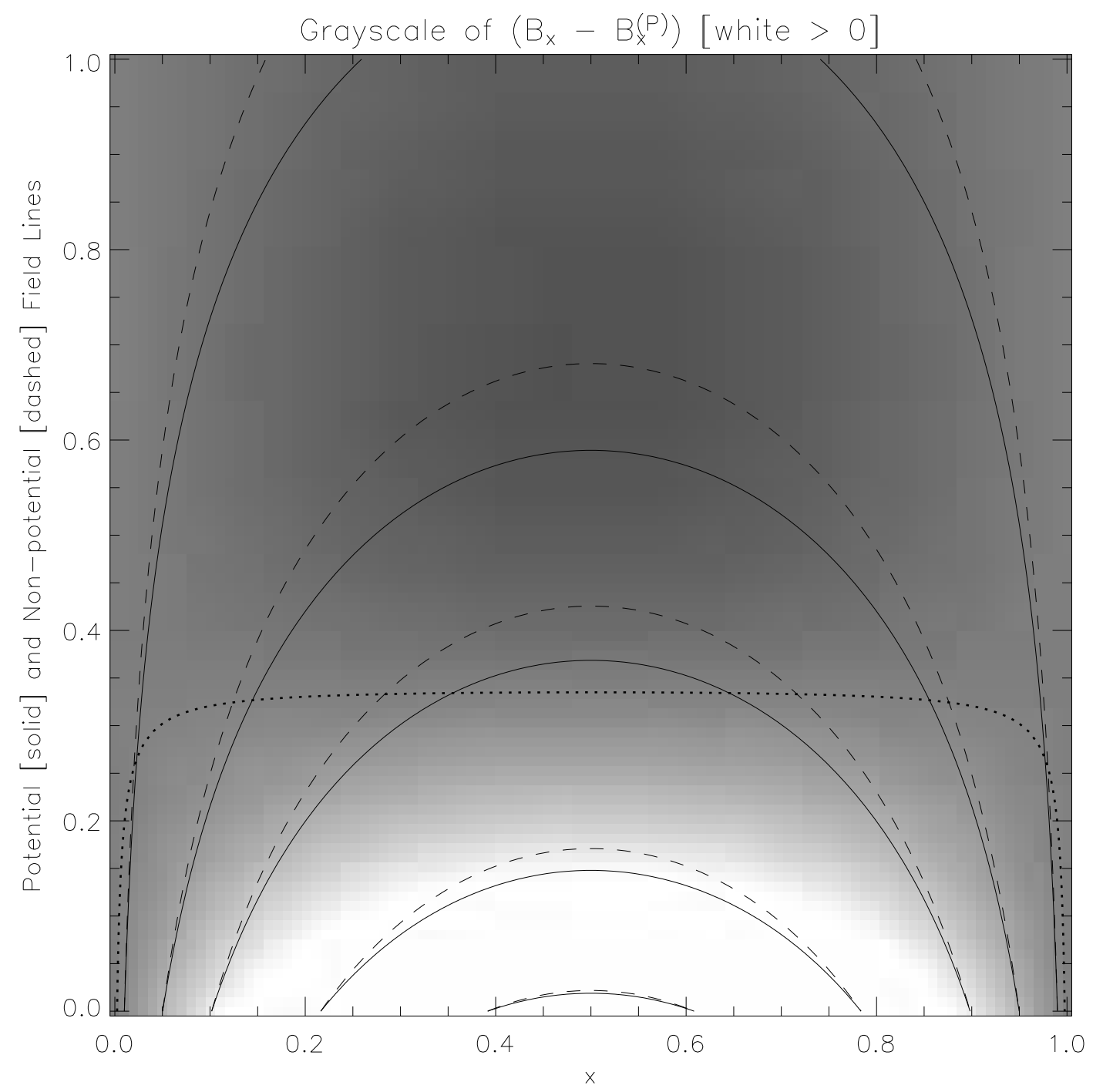

Fig. 3.- 